\title{
Ethiopian barley landraces: useful resistant sources to manage Barley yellow dwarf and other foliar diseases constraining productivity
}

\author{
Berhanu Bekele • Adane Abraham • \\ Safaa G. Kumari • Seid Ahmed • Chemeda Fininsa • \\ Abdurazak Yusuf
}

Accepted: 19 November 2018 /Published online: 8 July 2019

C) Koninklijke Nederlandse Planteziektenkundige Vereniging 2019

\begin{abstract}
One hundred sixty-five advanced lines were subjected to marker-assisted selection using Cleaved amplified polymorphic sequence-polymerase chain reaction (CAPS-PCR) primer against Barley yellow dwarf (BYD) resistant $Y d 2$ gene. In addition, phenotypic evaluation was employed to screen the advanced lines for multiple disease resistance. Effect of host growth rate and incubation period on BYD development was also assessed. Results of marker PCR showed that $Y d 2$ gene was detected in 96 (58.2\%) out of 165 accessions tested. Data on relationships of days to heading (DtH) and BYD symptoms development revealed that barley accessions having shorter DtH (66 days), BYD severity appeared low, and vice versa on accessions having longer DtH (79-83 days). Screening for multiple disease resistance gave interesting results. Among 11 accessions
\end{abstract}

B. Bekele

Ethiopian Institute of Agricultural Research, Ambo Plant

Protection Research Centre, Ambo, Ethiopia

B. Bekele $\cdot$ C. Fininsa $\cdot$ A. Yusuf

Haramaya University, Dire Dawa, Ethiopia

A. Abraham

Addis Ababa Science and Technology University, Addis Ababa, Ethiopia

S. G. Kumari $(\bowtie)$

International Centre for Agricultural Research in the Dry Areas

(ICARDA), Terbol Station, Zahla, Lebanon

e-mail: s.kumari@cgiar.org

S. Ahmed

ICARDA, Rabat, Morocco (with $Y d 2$ ) selected as the best for multiple diseases, 6 accessions [Chelia local, 4304-2(2), 4818(1.3), 18221(1), 1831-2-2(2), and 4915-1(1.2)] were highly resistant to leaf rust and net blotch, while other five lines had moderately resistant reaction. Study on the relationship of host resistance and incubation period showed that BYDV was detectable 5 days earlier in susceptible than in resistant lines. This can be used as an alternative method in preliminary screening of large number of genotypes against BYDV. From this study, it was evident that the identified resistant genotypes are a good resource for barley improvement program targeting BYDV and other important foliar pathogens. Barley breeders are encouraged to take advantage of this opportunity in producing multi-disease resistant barley genotypes in an agronomically enhanced genetic background in the country and beyond.

Keywords Landraces · Marker assisted selection (MAS) · Barley yellow dwarf virus (BYDV) · Yd2

\section{Introduction}

Barley (Hordeum vulgare L.) is among the most economically important cereals grown in Ethiopia. In terms of area coverage and total production, barley ranks 5th next to tef, maize, sorghum and wheat (CSA 2016). It covered over one million ha with a total production of 2.0 million tones, and productivity of $1.81 \mathrm{t}$ /ha. Biotic stress such as diseases take the lion share accounting for such low productivity. Ethiopia is an important primary and secondary gene 
center for many field crop species, including barley with wide genetic diversity (Adugna 2011; Harlan 1969; Vavilov 1951). Several traits, such as disease resistance to Barley yellow dwarf virus-PAV (BYDV-PAV), powdery mildew, leaf rust, loose smut, and Barley stripe mosaic virus (BSMV), and high lysine content were identified from barley germplasm originated from Ethiopia (Abebe 2006). The availability of resistance traits for various economically important diseases is an opportunity to be exploited to develop multiple disease resistance.

Breeding new varieties through conventional methods take up to 12 years and even then, the release of an improved variety cannot be guaranteed (Korzun 2003). The advent and applications of molecular marker technologies worldwide, however, is changing the scene significantly by speeding up the classical crop improvement program by assessing and/or introgression of rich gene pool that exists in the cultivated crops and their wild relatives. Molecular markers are of paramount importance in serving as chromosomal landmarks to trace the presence of a specific genomic region related to a useful trait during the breeding process. Consequently, they are helpful to accumulate multiple genes for resistance to specific pathogens within the same cultivar (gene pyramiding). Besides mapping and tagging genes, molecular markers have made it also possible to map and characterize the polygenes underlying quantitative traits in natural populations (QTL mapping). Molecular diagnostic methods based on polymerase chain reaction (PCR) technology, such as random amplified polymorphic DNA (RAPD), amplified fragment length polymorphism (AFLP) and simple sequence repeat microsatellite (SSR) emerged in the 1980s and early 1990, and their use to enhance plant breeding efforts has been described by many investigators (Dudley 1993; Paterson et al. 1991; Stuber et al. 1999).

Located on chromosome 3H (Schaller et al. 1964), the BYDV resistance gene ( $Y d 2$ ) was first identified by Rasmusson and Schaller (1959) in barleys originated from Ethiopia. Later, Collins et al. (1996) has accurately mapped and demonstrated that $\mathrm{Yd} 2$ gene co-segregated with RFLP markers Xwg889 and XY1p on the long arm of chromosome 3 , about $0.5 \mathrm{cM}$ from the centromere. Sequence data further showed that the Ylp alleles differ by a single nucleotide in barley with and without $Y d 2$. Such close linkage suggested to provide an opportunity to develop a strong marker for $Y d 2$ selection. Paltridge et al. (1998) and Jefferies et al. (2003) were also developed a co-dominant PCR-based marker designated
YLM. More recently, the other resistant gene 'Ryd3' was identified from barley and linked to the SSR markers HVM22, HVM14, HVM65, HVM74, Bmac0018 and Bmac0009 (Niks et al. 2004). Yd3 located on a different chromosome (chromosome $6 \mathrm{H}$ ) (Spaner et al., 1998; Ramsay et al., 2000) than Ryd2, and their location on a different chromosome suggested as an advantage to easily combine both genes in one cultivar to attain a higher level of BYD resistance than either alone. These resistant genes are currently widely used all over the world in barley improvement programs. Whilst both genes were identified from the Ethiopian barley landrace collections, they were not incorporated into elite barley cultivars.

Barley is challenged by the presence of complex of more than one disease that commonly occur in farmers' fields at the same time. Leaf rust (caused by Puccinia hordei Otth.) and net blotch (caused by Pyrenophora teres Drechsler) are, among others, the two major fungal diseases constraining barley production in major growing areas in Ethiopia (Ayele et al. 2008). Among cereal viruses, BYDV-PAV is causing significant impact on highland grown barley in Ethiopia (Agranovsky 1986; Bekele 1998; Bekele et al. 2001, 2003). The level of damage caused by these diseases necessitated development of efficient control measures. Since chemical application is not an economically or environmentally sound solution, the development of varieties with durable resistance is a priority for breeding programs. The aim of this investigation was: primarily, to screen barley accessions/landraces resistant to BYDV, using Yd2 linked marker (YLP) (Ford et al. 1998), and phenotypic evaluation for leaf rust and net blotch; secondly, to determine whether differences exist between lines with and without $Y d 2$ gene on virus incubation period and/or movement; and thirdly, to understand if there is any association between plant growth rate [days to heading (DtH)] and BYD severity.

\section{Materials and methods}

Evaluation of barley accessions to BYDV-PAV, leaf rust and net blotch

Plant materials and DNA extraction Plant materials used in this study was earlier selected and advanced from around 1400 barley landrace collections and released cultivars obtained from Ethiopian Biodiversity 
Institute (EBI) and Barley Improvement Department of Holetta Agricultural Research Centre (HARC) (PPRC 2012). The selected materials were further purified for "true to type" based on barley descriptors using morphological features (IPGRI 1994). A total of 165 genotypes, of which 143 barley landrace collections identified earlier at Plant Protection Research Center (PPRC) as promising lines against BYDV-PAV under field conditions and 22 released and elite candidates were evaluated using PCR marker (Yd2-linked marker) to determine whether the known BYDV-PAV resistance gene is involved in the field observed resistance reactions. Each genotype was planted in un-replicated, two row plots of $0.6 \mathrm{~m} \times 1 \mathrm{~m}=0.6 \mathrm{~m}^{2}$, with spacing of $0.3 \mathrm{~m}$ and $0.5 \mathrm{~m}$, between rows and plots, respectively. About $1 \mathrm{~g}$ barley plants were sampled at 1-2 leaf growth stages (11-12 Zadoks scale) (Zadoks et al. 1974) for DNA extraction. Total plant DNA was extracted using cetyl trimethyl ammonium bromide (CTAB) buffer as essentially described by Doyle and Doyle (1990) with the modification that samples were ground in liquid nitrogen using tissue layser. All other plants of each genotypes were allowed to grow until maturity to monitor development of BYD symptoms and other foliar diseases. To guarantee BYD development, cultivars "Gold (CI 1145)" and "HB-42" were planted at a uniform interval as a source of infection row one weak ahead of test genotypes. In addition, to supplement natural infection, viruliferous aphid vectors (Rhopalosiphum padi L.) reared at PPRC greenhouse were uniformly distributed on test plants to promote infection. Superimposed on the same barley materials, host response to leaf rust and net blotch were phenotypically evaluated. Data on heading date, BYD symptoms severity ( $0-9$ scale) (Schaller and Qualset, 1980) at booting stage (41-49 Zadoks scale) (Zadoks et al. 1974), and leaf rust (Pucinia hordei Otth.) and net blotch (Pyrenophora teres Drechs.) severity and plant response to infection were recorded twice at heading and flowering using modified Cobbs Scale (Peterson et al. 1948) (Table 1).

Polymerase chain reaction (PCR) analysis for $Y d 2$ gene PCR amplification of $Y d_{2}$ gene was performed in $15 \mu \mathrm{l}$ reaction volume containing $3 \mu \mathrm{l}$ Buffer, $8.85 \mu \mathrm{l} \mathrm{SdH}_{2} \mathrm{O}, 10$ pmole each primer $(0.5 \mu \mathrm{l}$ each), $0.15 \mu \mathrm{l}$ Taq polymerase and $2 \mu \mathrm{l}$ genomic DNA. PCR was run on a standard thermocycler with reaction conditions of $94{ }^{\circ} \mathrm{C}$ for $5 \mathrm{~min}$ followed by 35 cycles of $94{ }^{\circ} \mathrm{C}$ for $30 \mathrm{~s}, 53{ }^{\circ} \mathrm{C}$ for $1 \mathrm{~min}$ and
$72{ }^{\circ} \mathrm{C}$ for $2 \mathrm{~min}$, and a final extension step of $72{ }^{\circ} \mathrm{C}$ for $5 \mathrm{~min}$. The CAPS-PCR primers (Ford et al. 1998) used for the amplification of the $Y d 2$ gene were Y1p-MF (AATACAGGAATCTGTTGAAA GAA) and Y1p-MR (TCATCATGGCTCGG AGAAGGTGG). PCR products $(10 \mu \mathrm{l} /$ well, each) were separated by $2.5 \%$ agarose gel electrophoresis in $1 \mathrm{x}$ TAE (Tris-Acetate-EDTA) buffer stained with $5 \mu \mathrm{l}$ Redsafe $^{\mathrm{TM}}$ nucleic acid staining solution (20,000x) (iNtRON Biotechnology) and visualized using UV light transillumination. For reference and comparison of PCR products, 100 bp DNA size marker (iNtRON Biotechnology) was used along with the samples at $4 \mu \mathrm{l} /$ well.

Relationships of virus incubation period and host plant resistance

Plant materials and virus inoculum preparation Sixtyseven barley accessions (Table 2), randomly selected from 165 field selected advanced barleys, were evaluated in the greenhouse to determine association of BYDV-PAV incubation period or movement and host plant resistance. Based on the PCR results (above), both susceptible $(-Y d 2)$ and resistant $(+Y d 2)$ genotypes were included. All accessions were planted in pots in greenhouse at ambient room temperature. Culture of local BYDV-PAV isolate earlier identified at PPRC were used in this study. Similarly, for virus acquisition and inoculation, a local colony of $R$. padi was reared in greenhouse at ambient room temperature $\left(\sim 22{ }^{\circ} \mathrm{C}\right)$. Test plants were grown in pots of $23 \mathrm{~cm} \times 20 \mathrm{~cm}$, with each pot planted to six seeds for each treatment and maintained in the growth house with day temperature of $20-22{ }^{\circ} \mathrm{C}$. A $48 \mathrm{~h}$ acquisition and inoculation access periods adopted, and 10 viruliferous aphids per plant were used for inoculation feeding. All plants receiving different treatments were inoculated equally at $2-3$ leaf stages. Six treatments were tested, namely 2, 5, 10, 15, 20 and 25 days after inoculation (DAI).

Laboratory analysis using tissue blot immunoassay (TBIA) Plants of each treatment were tested by tissue blot immunoassay (TBIA) test as essentially described by Makkouk and Comeau (1994). Stems of test plants from each treatment were cut using new razor blades and blotted on nitrocellulose membrane $(\mathrm{NCM})$ and analyzed by the TBIA. A 
Table 1 Cleaved amplified polymorphic sequence-polymerase chain reaction (CAPS-PCR) amplification of $Y d 2$ gene and reaction of barley genotypes to Barley yellow dwarf virus (BYD), leaf rust and net blotch at Ambo, Ethiopia, 2015/2016 main cropping seasons

\begin{tabular}{|c|c|c|c|c|c|c|}
\hline No. & Accession & $\begin{array}{l}\text { Days to } \\
\text { heading }\end{array}$ & $Y d 2$ gene & $\begin{array}{l}\text { BYD symptoms } \\
\text { severity }(0-9)\end{array}$ & $\begin{array}{l}\text { Net blotch } \\
\text { severity }(\%)\end{array}$ & $\begin{array}{l}\text { Leaf rust severity } \\
(\%) \text { and response }\end{array}$ \\
\hline 1 & $1785-1-1$ & 66 & + & 0 & 25 & $7 \mathrm{MR}$ \\
\hline 2 & $3324-1-1(2)$ & 66 & + & 3 & 5 & $30 \mathrm{~S}$ \\
\hline 3 & $3412(2)$ & 66 & - & 2 & 65 & $45 \mathrm{~S}$ \\
\hline 4 & $3492-2(2)$ & 66 & + & 0 & 65 & $35 \mathrm{~S}$ \\
\hline 5 & $3675-1(4)$ & 66 & + & 0 & 25 & $25 \mathrm{~S}$ \\
\hline 6 & $4390-1(2.1)$ & 66 & - & 0 & 30 & $50 \mathrm{~S}$ \\
\hline 7 & $4390-2(1)$ & 66 & - & 0 & 15 & $85 \mathrm{~S}$ \\
\hline 8 & Shenen local(4) & 66 & + & 0 & 8 & $20 \mathrm{~S}$ \\
\hline 9 & $4199(2)$ & 67 & - & 0 & 55 & $50 \mathrm{~S}$ \\
\hline 10 & $1831-2-2(1)$ & 69 & + & 1 & 10 & $5 \mathrm{MR}$ \\
\hline 11 & $1836(1)$ & 69 & + & 0 & 5 & $20 \mathrm{MS}$ \\
\hline 12 & $1836(2)$ & 69 & - & 1 & 5 & $20 \mathrm{MS}$ \\
\hline 13 & $1838-1$ & 69 & - & 0 & 10 & $30 \mathrm{MS}$ \\
\hline 14 & $3167-2$ & 69 & - & 2 & 10 & $40 \mathrm{~S}$ \\
\hline 15 & $3324-1-1(1)$ & 69 & - & 0 & 3 & $40 \mathrm{~S}$ \\
\hline 16 & $3326-1-1$ & 69 & + & 0 & 45 & $25 \mathrm{~S}$ \\
\hline 17 & $3412(1)$ & 69 & + & 0 & 15 & $20 \mathrm{~S}$ \\
\hline 18 & $3492-2(1)$ & 69 & + & 0 & 45 & $40 \mathrm{~S}$ \\
\hline 19 & $3492-2(1.2)$ & 69 & - & 0 & 65 & $35 \mathrm{~S}$ \\
\hline 20 & $3492-2(3)$ & 69 & - & 2 & 30 & $40 \mathrm{~S}$ \\
\hline 21 & $3492-2(3.2)$ & 69 & - & 0 & 65 & $55 \mathrm{~S}$ \\
\hline 22 & $3675-1(1)$ & 69 & - & 0 & 65 & $20 \mathrm{MS}$ \\
\hline 23 & $3675-1(2)$ & 69 & - & 5 & 65 & $20 \mathrm{~S}$ \\
\hline 24 & $3675-1(3)$ & 69 & - & 2 & 35 & $60 \mathrm{~S}$ \\
\hline 25 & $3988-1(1)$ & 69 & - & 0 & 15 & $20 \mathrm{MS}$ \\
\hline 26 & $4075-2-3(1)$ & 69 & - & 0 & 20 & $30 \mathrm{~S}$ \\
\hline 27 & $4075-2-3(3)$ & 69 & - & 0 & 10 & $45 \mathrm{~S}$ \\
\hline 28 & 4199(1) & 69 & - & 2 & 30 & $65 \mathrm{~S}$ \\
\hline 29 & 4199(3) & 69 & - & 0 & 20 & $50 \mathrm{~S}$ \\
\hline 30 & $4228-1-2(1)$ & 69 & - & 0 & 15 & $45 \mathrm{~S}$ \\
\hline 31 & $4228-1-2(2)$ & 69 & + & 1 & 25 & $35 \mathrm{~S}$ \\
\hline 32 & $4228-1-2(3)$ & 69 & + & 0 & 25 & $45 \mathrm{~S}$ \\
\hline 33 & $4228-1-2(4)$ & 69 & - & 0 & 30 & $50 \mathrm{~S}$ \\
\hline 34 & $4228-2(1)$ & 69 & + & 0 & 20 & $50 \mathrm{~S}$ \\
\hline 35 & $4228-2(2)$ & 69 & + & 2 & 15 & $65 \mathrm{~S}$ \\
\hline 36 & $4238-2(1)$ & 69 & - & 2 & 30 & $60 \mathrm{~S}$ \\
\hline 37 & $4243-1-1$ & 69 & + & 0 & 3 & $25 \mathrm{~S}$ \\
\hline 38 & $4253(1)$ & 69 & + & 0 & 40 & $30 \mathrm{~S}$ \\
\hline 39 & $4253(2)$ & 69 & + & 1 & 15 & $45 \mathrm{~S}$ \\
\hline 40 & $4264(2)$ & 69 & - & 0 & 15 & $70 \mathrm{~S}$ \\
\hline 41 & $4290-2(2)$ & 69 & - & 0 & 25 & $60 \mathrm{~S}$ \\
\hline 42 & $4291-1(1.2)$ & 69 & + & 0 & 35 & $40 \mathrm{~S}$ \\
\hline 43 & $4300-1(2)$ & 69 & - & 0 & 25 & $40 \mathrm{~S}$ \\
\hline
\end{tabular}


Table 1 (continued)

\begin{tabular}{|c|c|c|c|c|c|c|}
\hline No. & Accession & $\begin{array}{l}\text { Days to } \\
\text { heading }\end{array}$ & $Y d 2$ gene & $\begin{array}{l}\text { BYD symptoms } \\
\text { severity }(0-9)\end{array}$ & $\begin{array}{l}\text { Net blotch } \\
\text { severity }(\%)\end{array}$ & $\begin{array}{l}\text { Leaf rust severity } \\
(\%) \text { and response }\end{array}$ \\
\hline 44 & $4300-1(3)$ & 69 & + & 0 & 15 & $85 \mathrm{~S}$ \\
\hline 45 & $4300-1(4)$ & 69 & + & 0 & 15 & $50 \mathrm{~S}$ \\
\hline 46 & 4312 & 69 & + & 1 & 20 & $50 \mathrm{~S}$ \\
\hline 47 & $4304-2(1)$ & 69 & + & 0 & 15 & $45 \mathrm{~S}$ \\
\hline 48 & $4390-1(1.1)$ & 69 & - & 4 & 20 & $35 \mathrm{~S}$ \\
\hline 49 & $4390-1(1.2)$ & 69 & - & 1 & 10 & $35 \mathrm{~S}$ \\
\hline 50 & $4390-1(1.3)$ & 69 & + & 2 & 15 & $55 \mathrm{~S}$ \\
\hline 51 & $4418-2-2(1)$ & 69 & - & 0 & 15 & $30 \mathrm{~S}$ \\
\hline 52 & $4756(1)$ & 69 & - & 2 & 30 & $50 \mathrm{~S}$ \\
\hline 53 & $4756(2)$ & 69 & + & 0 & 15 & $45 \mathrm{~S}$ \\
\hline 54 & $4756(4)$ & 69 & + & 0 & 15 & $90 \mathrm{~S}$ \\
\hline 55 & $4765-2$ & 69 & + & 0 & 25 & $40 \mathrm{~S}$ \\
\hline 56 & $4767-1-1$ & 69 & + & 2 & 15 & $30 \mathrm{~S}$ \\
\hline 57 & $4806-1(1)$ & 69 & + & 1 & 15 & $20 \mathrm{~S}$ \\
\hline 58 & $4841-2(1.3)$ & 69 & + & 0 & 20 & $5 \mathrm{MR}$ \\
\hline 59 & 4883 & 69 & + & 0 & 20 & $25 \mathrm{~S}$ \\
\hline 60 & 4915-1(1.1) & 69 & + & 0 & 15 & $80 \mathrm{~S}$ \\
\hline 61 & $64,016-1-2$ & 69 & + & 1 & 8 & $45 \mathrm{~S}$ \\
\hline 62 & $64,105(1.1)$ & 69 & + & 4 & 15 & $85 \mathrm{~S}$ \\
\hline 63 & $64,106-2-1$ & 69 & + & 3 & 2 & $75 \mathrm{~S}$ \\
\hline 64 & $64,178(1.1)$ & 69 & - & 5 & 5 & $30 \mathrm{~S}$ \\
\hline 65 & $64,223(2)$ & 69 & + & 0 & 15 & $25 \mathrm{~S}$ \\
\hline 66 & $64,223(3)$ & 69 & + & 3 & 5 & $30 \mathrm{~S}$ \\
\hline 67 & $64,226-1$ & 69 & + & 4 & 7 & $40 \mathrm{~S}$ \\
\hline 68 & HB-42(11) & 69 & + & 4 & 10 & $30 S$ \\
\hline 69 & IBON 170/03 & 69 & + & 2 & 1 & $5 \mathrm{R}$ \\
\hline 70 & Chelia Local (1) & 69 & + & 3 & $<1$ & $<1 \mathrm{R}$ \\
\hline 71 & $4238-2(2)$ & 70 & - & 2 & 15 & $60 \mathrm{~S}$ \\
\hline 72 & $4239-2-1$ & 70 & - & 2 & 5 & $35 \mathrm{MS}$ \\
\hline 73 & $4239-2-2(1)$ & 70 & - & 0 & 5 & $25 \mathrm{~S}$ \\
\hline 74 & 4385 & 70 & - & 2 & 30 & $30 \mathrm{~S}$ \\
\hline 75 & $4264(1)$ & 71 & + & 0 & 15 & $65 \mathrm{~S}$ \\
\hline 76 & $4290-2(1)$ & 71 & + & 0 & 65 & $85 \mathrm{~S}$ \\
\hline 77 & $4291-1(2.1)$ & 71 & + & 0 & 40 & $45 \mathrm{~S}$ \\
\hline 78 & $4291-1(2.2)$ & 71 & + & 0 & 50 & $30 \mathrm{~S}$ \\
\hline 79 & $4291-1(3.1)$ & 71 & - & 0 & 50 & $45 \mathrm{~S}$ \\
\hline 80 & $4300-1(1)$ & 71 & + & 0 & 15 & $60 \mathrm{~S}$ \\
\hline 81 & $4304-2(3)$ & 71 & + & 1 & 15 & $45 \mathrm{~S}$ \\
\hline 82 & $4382-1$ & 71 & + & 2 & 10 & $90 \mathrm{~S}$ \\
\hline 83 & $4388(2)$ & 71 & - & 2 & 15 & $25 \mathrm{~S}$ \\
\hline 84 & $4390-2(2)$ & 71 & + & 1 & 15 & $25 \mathrm{MS}$ \\
\hline 85 & $4415(1)$ & 71 & - & 2 & 5 & $90 \mathrm{~S}$ \\
\hline 86 & $4415(2)$ & 71 & - & 4 & 35 & $35 \mathrm{~S}$ \\
\hline 87 & $4418-2-2(2)$ & 71 & - & 0 & 30 & $30 \mathrm{~S}$ \\
\hline
\end{tabular}


Table 1 (continued)

\begin{tabular}{|c|c|c|c|c|c|c|}
\hline No. & Accession & $\begin{array}{l}\text { Days to } \\
\text { heading }\end{array}$ & $Y d 2$ gene & $\begin{array}{l}\text { BYD symptoms } \\
\text { severity }(0-9)\end{array}$ & $\begin{array}{l}\text { Net blotch } \\
\text { severity }(\%)\end{array}$ & $\begin{array}{l}\text { Leaf rust severity } \\
(\%) \text { and response }\end{array}$ \\
\hline 88 & $4530-1(1)$ & 71 & - & 2 & 15 & $55 \mathrm{~S}$ \\
\hline 89 & $4530-1(2)$ & 71 & + & 0 & 20 & $55 \mathrm{~S}$ \\
\hline 90 & $4756(3)$ & 71 & + & 0 & 10 & $60 \mathrm{~S}$ \\
\hline 91 & $4769-1-2(1.1)$ & 71 & + & 0 & 12 & $15 \mathrm{MS}$ \\
\hline 92 & $4769-1-2(2)$ & 71 & + & 0 & 8 & $35 \mathrm{MS}$ \\
\hline 93 & $4769-2$ & 71 & + & 0 & 15 & $10 \mathrm{MS}$ \\
\hline 94 & $4773-2$ & 71 & + & 1 & 25 & $55 \mathrm{~S}$ \\
\hline 95 & $4784-1(1.1)$ & 71 & - & 0 & 8 & $40 \mathrm{~S}$ \\
\hline 96 & $4784-1(2.1)$ & 71 & - & 2 & 10 & $45 \mathrm{~S}$ \\
\hline 97 & $4784-1(2.2)$ & 71 & + & 0 & 12 & $20 \mathrm{~S}$ \\
\hline 98 & $4806-1(1)$ & 71 & + & 0 & 8 & $25 \mathrm{~S}$ \\
\hline 99 & $4818(1.1)$ & 71 & + & 2 & 65 & $25 \mathrm{~S}$ \\
\hline 100 & $4818(1.2)$ & 71 & ++ & 1 & 50 & $40 \mathrm{~S}$ \\
\hline 101 & $4818(1.3)$ & 71 & + & 2 & 2 & $1 \mathrm{R}$ \\
\hline 102 & $4818(2)$ & 71 & ++ & 0 & 50 & $45 \mathrm{~S}$ \\
\hline 103 & $4823-3(1.1)$ & 71 & + & 1 & 10 & $60 \mathrm{~S}$ \\
\hline 104 & $4823-3(1.2)$ & 71 & + & 1 & 10 & $45 \mathrm{MS}$ \\
\hline 105 & $4833-2$ & 71 & - & 4 & 10 & $60 \mathrm{~S}$ \\
\hline 106 & $4841-2(1.1)$ & 71 & + & 4 & 8 & $5 \mathrm{MR}$ \\
\hline 107 & $4981-1$ & 71 & + & 7 & 12 & $20 \mathrm{~S}$ \\
\hline 108 & $64,068-1$ & 71 & - & 2 & 25 & $65 \mathrm{~S}$ \\
\hline 109 & $64,105(1.2)$ & 71 & + & 2 & 10 & $80 \mathrm{~S}$ \\
\hline 110 & $64,178(1.2)$ & 71 & + & 1 & 7 & $85 \mathrm{~S}$ \\
\hline 111 & $64,217-2(1.1)$ & 71 & + & 3 & 7 & $40 \mathrm{~S}$ \\
\hline 112 & $64,223(1)$ & 71 & + & 1 & 25 & $45 \mathrm{~S}$ \\
\hline 113 & Gold (CI1145) & 71 & + & 4 & 15 & $55 \mathrm{~S}$ \\
\hline 114 & Shenen local (2) & 71 & + & 0 & 10 & $20 \mathrm{~S}$ \\
\hline 115 & HB-1307 (EH-1700/F71.B1.63) & 71 & + & 2 & 8 & $30 \mathrm{~S}$ \\
\hline 116 & Cross \# 41/98 & 71 & + & 5 & $<1$ & $2 \mathrm{R}$ \\
\hline 117 & Miscal-21 & 71 & + & 3 & 2 & $2 \mathrm{R}$ \\
\hline 118 & Bekoji 1(EH1293/F2-18B-11-1-14-18) & 71 & - & 5 & 2 & $2 \mathrm{R}$ \\
\hline 119 & Gold (CI1145) & 71 & + & 5 & 2 & $20 \mathrm{MS}$ \\
\hline 120 & Chelia local (2) & 71 & - & 5 & $<1$ & $5 \mathrm{MR}$ \\
\hline 121 & $4769-1-2(1.2)$ & 71 & + & 1 & 10 & $30 \mathrm{~S}$ \\
\hline 122 & $4769-1-2(2)$ & 71 & - & 1 & 2 & $35 \mathrm{~S}$ \\
\hline 123 & $4841-2(1.1)$ & 71 & - & 2 & 5 & $85 \mathrm{~S}$ \\
\hline 124 & $4841-2(1.2)$ & 71 & + & 2 & 5 & 85 \\
\hline 125 & $4784-1(2)$ & 71 & + & 2 & 2 & $10 \mathrm{~S}$ \\
\hline 126 & $4806-1(2)$ & 71 & - & 0 & 85 & $5 \mathrm{MS}$ \\
\hline 127 & $64,217-2(1.2)$ & 71 & - & 2 & 8 & $10 \mathrm{~S}$ \\
\hline 128 & $64,217-2(2)$ & 72 & + & 2 & 7 & $85 \mathrm{~S}$ \\
\hline 129 & Shenen local (1) & 72 & + & 1 & 10 & $20 \mathrm{~S}$ \\
\hline 130 & 1729.2 & 72 & + & 2 & 5 & $40 \mathrm{MS}$ \\
\hline 131 & 1820 & 73 & ++ & 1 & 10 & $5 \mathrm{MR}$ \\
\hline
\end{tabular}


Table 1 (continued)

\begin{tabular}{|c|c|c|c|c|c|c|}
\hline No. & Accession & $\begin{array}{l}\text { Days to } \\
\text { heading }\end{array}$ & $Y d 2$ gene & $\begin{array}{l}\text { BYD symptoms } \\
\text { severity }(0-9)\end{array}$ & $\begin{array}{l}\text { Net blotch } \\
\text { severity }(\%)\end{array}$ & $\begin{array}{l}\text { Leaf rust severity } \\
(\%) \text { and response }\end{array}$ \\
\hline 132 & $3988-1(2)$ & 73 & - & 2 & 10 & $25 \mathrm{MS}$ \\
\hline 133 & $4243-1-2(1)$ & 73 & - & 0 & 3 & $25 \mathrm{MS}$ \\
\hline 134 & $3988-1(3)$ & 74 & - & 5 & 5 & $30 \mathrm{~S}$ \\
\hline 135 & $4239-2-2(2)$ & 74 & + & 0 & 1 & $10 \mathrm{MR}$ \\
\hline 136 & Shenen local (3) & 74 & - & 4 & 3 & $35 \mathrm{~S}$ \\
\hline 137 & EH 1847 /F4.2P.5.2 (BEA/IBON 64/91) & 74 & - & 5 & 1 & $2 \mathrm{R}$ \\
\hline 138 & $4915-1(1.2)$ & 74 & + & 1 & 10 & $5 \mathrm{R}$ \\
\hline 139 & 4915-1(1.3) & 74 & + & 1 & 10 & $10 \mathrm{MR}$ \\
\hline 140 & $3713-2$ & 76 & - & 0 & 3 & $45 \mathrm{~S}$ \\
\hline 141 & $1818-1(1)$ & 79 & - & 0 & 10 & $1 \mathrm{R}$ \\
\hline 142 & $1822-1(1)$ & 79 & + & 0 & 5 & $1 \mathrm{R}$ \\
\hline 143 & $1822-1(2)$ & 79 & - & 2 & 5 & $5 \mathrm{MR}$ \\
\hline 144 & $4046-1$ & 79 & - & 1 & 3 & $35 \mathrm{~S}$ \\
\hline 145 & $4075-2-3(2)$ & 79 & - & 5 & 3 & $3 R$ \\
\hline 146 & HB-42(1) & 79 & + & 7 & $<1$ & $2 \mathrm{R}$ \\
\hline 147 & HB-42(2) & 79 & - & 8 & 1 & $1 \mathrm{R}$ \\
\hline 148 & $4243-1-2(2)$ & 79 & - & 5 & 2 & $20 \mathrm{~S}$ \\
\hline 149 & $4304-2(2)$ & 79 & + & 7 & 1 & $1 \mathrm{R}$ \\
\hline 150 & Holker & 80 & + & 4 & 2 & $1 \mathrm{R}$ \\
\hline 151 & HB-42(3) & 83 & + & 5 & 5 & $2 \mathrm{R}$ \\
\hline 152 & HB-42(4) & 83 & - & 7 & 1 & $<1 \mathrm{R}$ \\
\hline 153 & $1831-2-2(2)$ & 83 & + & 0 & 10 & $2 \mathrm{R}$ \\
\hline 154 & $1818-1(2)$ & 83 & + & 0 & 10 & $5 \mathrm{MR}$ \\
\hline 155 & HB-42(5) & 83 & + & 7 & 1 & $2 \mathrm{R}$ \\
\hline 156 & $\mathrm{HB}-42(6)$ & 83 & + & 7 & $<1$ & $2 \mathrm{R}$ \\
\hline 157 & HB-42(7) & 83 & + & 7 & $<1$ & $1 \mathrm{R}$ \\
\hline 158 & $\mathrm{HB}-42(8)$ & 83 & - & 5 & 10 & $5 \mathrm{R}$ \\
\hline 159 & HB-42(9) & 83 & - & 5 & 2 & $3 R$ \\
\hline 160 & HB-42(10) & 83 & - & 8 & 1 & $1 \mathrm{R}$ \\
\hline 161 & $4388(1)$ & 83 & - & 5 & 3 & $75 \mathrm{~S}$ \\
\hline 162 & EH 1493/F6.32H.3 & 83 & + & 7 & 1 & $1 \mathrm{R}$ \\
\hline 163 & Ardu 12 & 83 & + & 5 & 1 & $1 \mathrm{R}$ \\
\hline 164 & $4784-1(1)$ & 83 & - & 7 & $<1$ & $3 \mathrm{MR}$ \\
\hline 165 & $4291-1(1.1)$ & 88 & + & 0 & 10 & $50 \mathrm{~S}$ \\
\hline
\end{tabular}

Accessions were arranged in ascending order, with those having similar days to head (DtH) were collected together to simplify comparison; \pm , Presence/absence of $Y d 2$ gene as detected by Ylp marker; BYD severity, assessed based on 0-9 scale (Schaller and Qualset, 1980); CI, Cereal introduction/ plant introduction accession number assigned by USDA; 0-85, percentage disease severity; R, Resistant; MR, moderately resistant; MS, moderately susceptible; S, Susceptible phenotype

1:1000 dilution of the BYDV-PAV polyclonal antibodies (=BYDV-B; Cat No. 140112, Bioreba, Switzerland) was used as the primary antibody. Goat anti-rabbit antibody conjugated to alkaline phosphatase was used as the probe antibody. Test plants considered positive based on the development of purplish to brown granules in the phloem tissue of the samples following the TBIA test. 


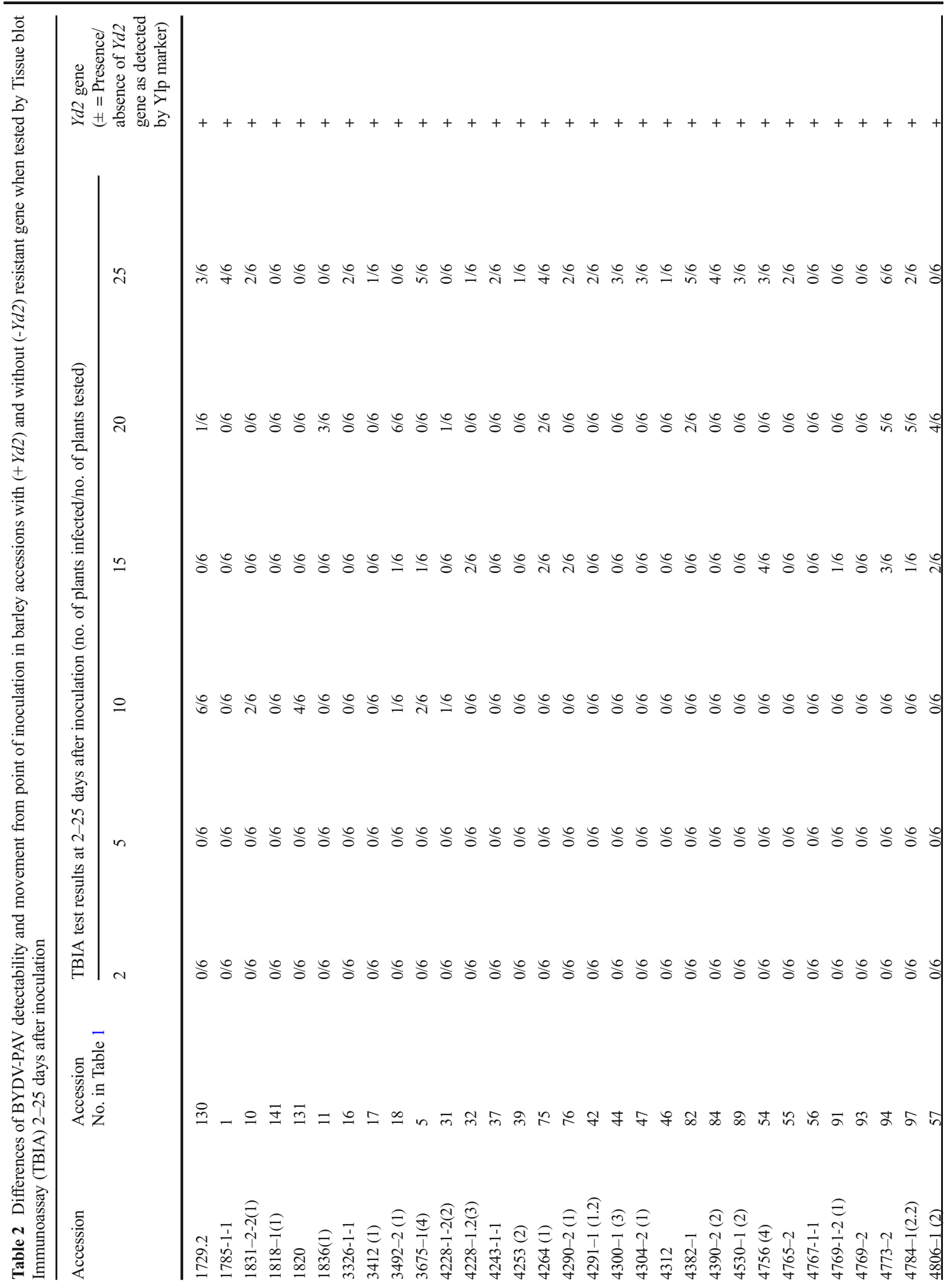




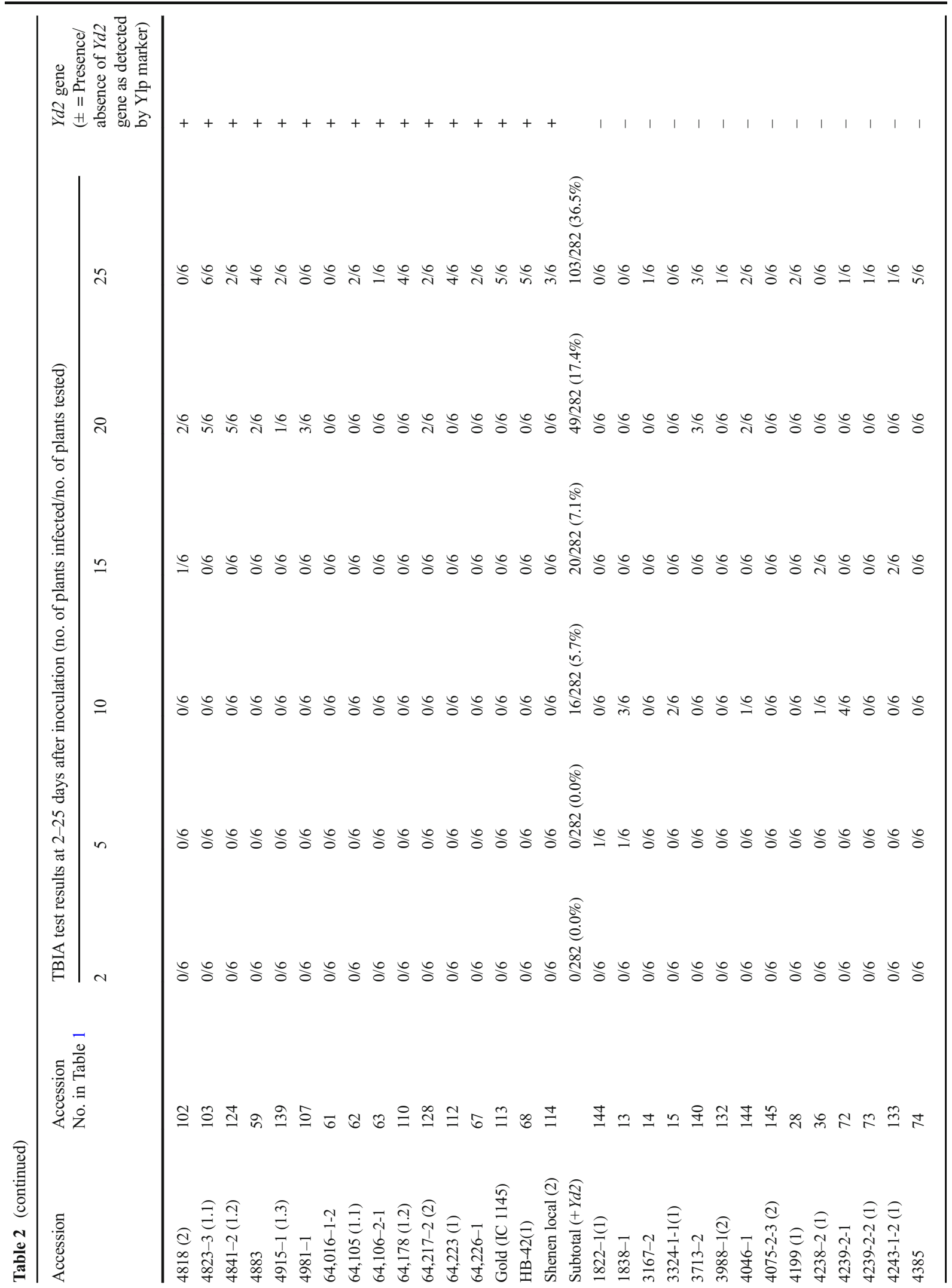




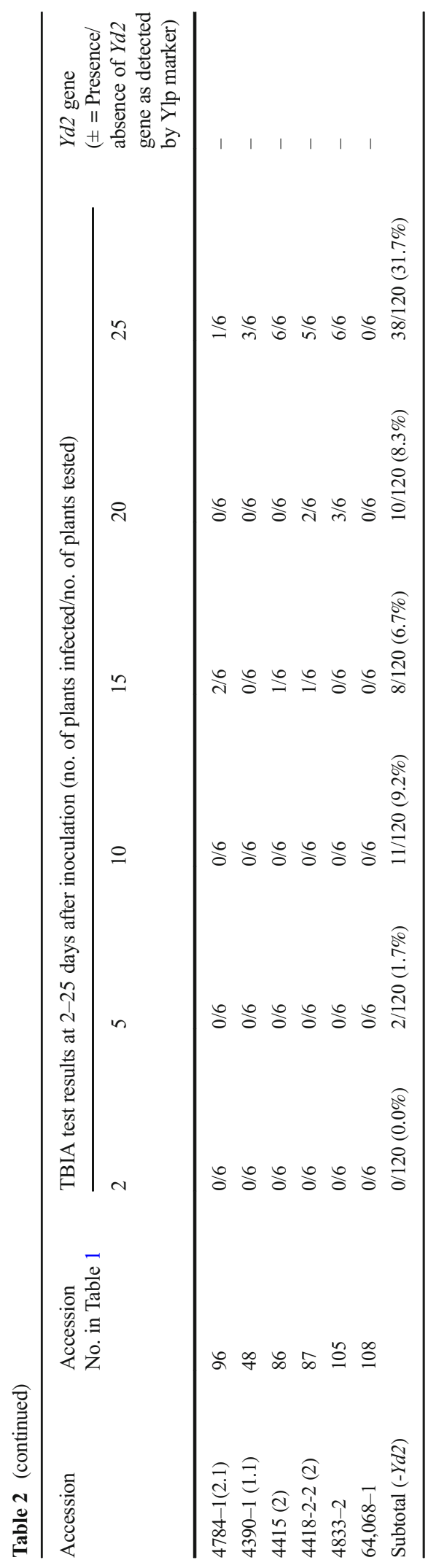

\section{Results}

Evaluation of barley accessions to BYDV-PAV, leaf rust and net blotch

Using the PCR-CAPS primers, expected product size (PS) (331 bp) related to Yd2 gene was amplified. Out of 165 accessions tested against the $Y d 2$-linked markers, $96(58.2 \%)$ of the tested genotypes were confirmed to contain the $Y d 2$ resistance gene (Table 1). Results of PCR, electrophoresis and UV visualization of purified 48 barley genotypes are shown in Fig. 1.

Data on the association of DtH and BYD symptoms severity was collected (Table 1) to compare if there were differential reactions among accessions. On barley accessions having short DtH (66 days) the disease severity appeared low, and vice versa on accessions having longer DtH (79-83 days). On the basis of DtH and disease symptoms severity score, all the genotypes can roughly be placed in two categories: (i) lines having no BYD symptoms or mild or medium with DtH from 66 to 76 days, and (ii) lines having medium to high symptoms with $\mathrm{DtH}$ from 79 to 83 days. At DtH from 66 to 76 days, symptomless lines were recorded in lines both with and without $Y d 2$ genes, while all symptomless lines at 79-83 DtH were only those lines with $Y d 2$ gene.

Phenotypic data collected to screen for multiple disease resistant barley showed that 11 lines that contained $Y d 2$ gene had a disease reaction ranged from highly resistant to moderate (Table 1) for leaf rust and net blotch. Among 11 lines selected as having good level of multiple disease resistance, 6 accessions that showed highest level of reactions were Chelia local, 4304-2(2), 4818(1.3), 1822-1(1), 1831-2-2(2), and 4915-1(1.2). These lines had disease severity and response ranging from $\angle 1 R$ to $5 \mathrm{R}$ and $\angle 1 \mathrm{R}$ to $10 \mathrm{MR}$, respectively, for leaf rust and net blotch. Despite all of them contained $Y d 2$ gene, 2 accessions [1822-1(1) and 1831-2-2(2)] did not develop any BYD-like symptoms, 2 developed low level of yellowing symptom, 1 medium and 1 highest symptoms such as yellowing and stunting. The other 5 accessions [4841-2(1.1), 4915-1(1.3), 1818-1(2), 1820 and 1831-2-2(1)] had moderate level of resistance, with medium (1 accession), low (3 accession) and no (1 accession) BYD symptom developed. Other four accessions (18181(1), 1822-1(2), 4075-2-3(2) and 4784-1(1) were negative to $Y d 2$ gene, but had low severity, and MR and $\mathrm{R}$ reactions to leaf rust and net blotch. 


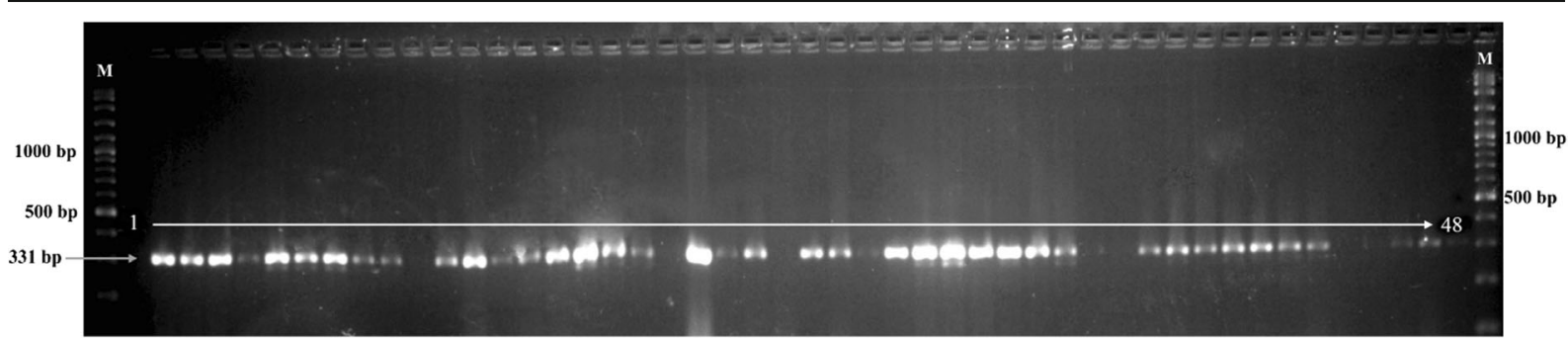

Fig. 1 PCR amplifications of $Y d 2$ gene (PS = $331 \mathrm{bp}$ ) conferring resistance to BYDVs from 48 barley accessions. No. $1-48=$ tested lines, $M=100$ bp DNA size ladder. Accession No. of tested lines (1 to 48$)$, in order, are: 4784(2.1), 4784(2-2), 4806-1(1), 48061(2), 4818(1.1), 4818(1.2), 4818(1.3), 4818(2), 4823-3(1.1), 4823-3(1.2), 4833-2, 4841-2(1.1), 4841-2(1.2), 4883, 49151(1.1), 4915-1(1.2), 4915-1(1.3), 4981-1, 64,016-1-2, 64,068-

Relationships of virus incubation period and host plant resistance

When barley plants containing resistance gene $(+Y d 2)$ was inoculated with BYDV-PAV at three leaf stage were tested by TBIA at 2, 5, 10, 15, 20, 25 DAI, BYDV was first detected at 10 DAI. The number of lines infected increased relatively proportionally from 10 DAI to 25 DAI (Table 2). Whilst, when 20 barley accessions without resistance gene $(-Y d 2)$ were tested by TBIA, BYDVPAV was detected 5 DAI. BYDV-PAV was not detectable in barley accessions with $(+Y d 2)$ or without $(-Y d 2)$ resistance gene 2 DAI. BYDV-PAV could not be recovered from any of the 6 treatments (DAI) in 4 accessions with (+Yd2) (accessions 1818-1, 4767-1-1, 4769-1-2 and 64,016-1-2) and two accessions without (-Yd2) (accessions 4075-2-3 and 64,068-1). In general, BYDV-PAV detectability increased from 10 DAI to 25 DAI in lines/accessions with $Y d 2$ gene, although it was inconsistently increased with the highest being recorded at $25 \mathrm{DAI}$, followed by $10 \mathrm{DAI}$ (Table 2).

\section{Discussion}

Ethiopian barley is considered highly diversified (Harlan 1969; Vavilov 1951) and landraces form the major genetic resources of cultivated barley in the country having several traits of agronomic importance (Abebe 2006). The two BYDV-PAV resistant major genes $(Y d 2$ and $Y d 3)$, for instance, exclusively identified from the Ethiopian barley (Niks et al. 2004; Rasmusson and Schaller 1959) and have since been transferred to several spring and winter barley cultivars and breeding
1, 64,105(1.1), 64,105(1.2), 64,106-2-1, 64,178(1.1), 64,178(1.2), 64,217.2(1), 64,217.2(2), 64,223(1), 64,223(2), 64,223(3), 64,226-1, Gold, HB-42, Shenen local(1), Shenen local(2), Shenen local(3), Shenen local(4), HB-1307, Cross 41/98, EH 1493, Ardu 12, Holker, Mis-21, Bekoji 1, EH 1847, IBON 170/03, Local, and Gold

lines across the Globe (Burnett et al. 1995). However, this potential had not yet been exploited in barley improvement programs in the country. In this study, $Y d 2$ gene was identified for the first time in significant proportion $(58.2 \%)$ of barley genotypes tested using marker PCR (Table 1). This proportion is very high, and in agreement with the findings of Qualset (1975) who reported that the Ethiopian barley collections from high elevations had a much higher frequency of BYD resistance than barleys from the lower areas. The fact that barley collections used in this study were from higher altitudes between 2500 and 3300 masl, and BYD severity and incidence in Ethiopia was more pronounced at same altitude range (Agranovsky 1986; Agranovsky et al. 1985; Bekele et al. unpublished; Bekele et al. 2001, 2003; Yusuf et al. 1992) explains the high proportion of BYDV resistant genotypes from Ethiopia. Based on this fact it is likely to assume that the ecological conditions at higher elevations in Ethiopia are more conducive for both the viruses and their local clones of respective aphid vectors for BYD epiphytotic development, which in turn exerted natural selection pressure geared up toward resulting abundant resistant phenotypes. Hence, this research findings further supported the fact that Ethiopia is rich gene pool for barley where BYDV resistant gene is readily identified from landrace collections and genetic resistance can be easily employed to reduce the effects of the disease. BYD is currently an economic disease of high land grown elite barleys, and the identified resistant genotypes are a good resource for future barley improvement program targeting BYDVs in Ethiopia. As shown in Table 1, some $Y d 2$ possessing accessions had higher BYD severity than the genotypes that lack this gene. This result 
may be interpreted in terms of occurrence of a resistance reaction called 'Tolerance' that permits plants to withstand high disease pressure with low yield loss.

A study to monitor the association of days to heading and development of BYD in accessions with $(+Y d 2)$ and without $(-Y d 2)$ resistant gene revealed that severe disease symptoms developed in lines having longer days to heading than lines having shorter days to heading. At 83 and $88 \mathrm{DtH}$, severe BYD symptoms developed in lines both with $(+Y d 2)$ and without $(-Y d 2)$ resistant gene. However, the proportion $(47 \%)$ of lines having severe symptoms was higher for those lines without resistant gene $(-Y d 2)$. In most of the lines having DtH of 66-69 (42\%), the symptoms were mild or absent. The general trend showed that as the DtH increased from 66 (shortest) to 88 (longest), disease severity and incidence increased proportionally, regardless of whether the resistant gene $(Y d 2)$ is present or not, assuming that the environment is similar. Two possible explanations can be suggested: (i) growth rate may affect the expression of BYD symptoms; or (ii) the level of tolerance conferred by $Y d 2$ gene may be modified by host genotype and environmental factors. Hayes et al. (1971) found significant positive correlation between levels of tolerance expressed by the parents and progeny with growth rate, suggesting that environmental or genetic factors which delay heading diminish the expression of BYDV tolerance. Similarly, research results of Jones and Catherall (1970) suggested that barley varieties were most tolerant to infection with barley yellow dwarf virus (BYDV) when they grew rapidly, whether the rate of growth was determined by manipulation of the environment or by the innate genetic constitution of the host. Having experienced difficulty in recovering late maturing segregates, Jones and Catherall (1970) suggested that the $Y d 2$ gene operates by retarding virus multiplication, thus allowing the virus to reach higher concentrations over a longer period of time.

In an attempt to adopt affordable and cheaper alternative methods of evaluation to differentiate resistant and susceptible barley genotypes using TBIA showed that BYDV-PAV was readily detectable five days after inoculation in stems of susceptible (-Yd2) than resistant $(+Y d 2)$ lines. This finding is in agreement with reports of previous studies on other crops that the relative restriction of virus movement in resistant cultivars has been documented for a number of sap transmissible viruses such as Cucumber mosaic virus (CMV) in pepper (Doufour et al. 1989; Nono-Womdim et al. 1991) and Maize dwarf mosaic virus (MDMV) in maize (Law et al. 1989). Previously, Jensen (1973) also reported that BYDV moves from an inoculated leaf more readily to roots in susceptible than in resistant cereals. Similarly, Makkouk and Ghulam (1992) reported differences in the rate of BYDV movement from the inoculated leaf to the root in resistant and susceptible barley genotypes. Makkouk et al. (1994) indicated that multiplication and/ or movement of barley yellow dwarf virus was retarded in resistant (R) than in susceptible (S) barley genotypes. They showed that DAS-ELISA testing of root extracts from 41 genotypes three or four days after inoculation at the one leaf stage resulted in reliable differentiation of susceptible from resistant plants. Further testing of barley plants inoculated at the two or three leaf stage using tissue-blot ELISA on nitrocellulose membranes revealed that the virus was detected 4-6 days after inoculation in the phloem vessels of the growing points of the susceptible genotypes, but not of resistant genotypes, indicating the method is rapid and inexpensive technique for screening for BYDV resistance in barley. It is to be recalled from Table 2 that BYDV-PAV was not uniformly and consistently recovered from TBIA testing five DAI and onwards among most accessions without resistant gene $(-Y d 2)$, and yet the virus was not recovered in two such lines. In the case of barley accessions with resistant gene $(+Y d 2)$, BYDV-PAV was recovered most frequently at $25 \mathrm{DAI}$, supporting the report that the $Y d 2$ resistant gene suppress/retard virus movement and/or multiplication. As in the case of accessions without $Y d 2$, BYDVPAV was not recovered from four lines. Absence of positive TBIA result in this case may be attributed to either inherent genetic background of the host that completely resist the virus conferred by the same gene or other gene(s) (major and minor) operating concurrently or infection escape or the efficiency of TBIA in detect low virus titer. Similarly, absence of positive TBIA reactions in two of $Y d 2$ negative barley accessions may also imply the presence of other effective gene(s) other than $\mathrm{Yd} 2$ or infection escape due to lack of uniform application of viruliferous aphid vectors. In general, as in the case of previous studies, the result based on virus movement gave good indications in selecting promising barley lines in preliminary screening. Thus, to use the method solely to develop BYDV resistant genotypes, supporting data such as yield and associated agronomic traits may be taken into consideration.

It is interesting to note that some barley genotypes found resistant to BYD were also resistant to leaf rust and 
net blotch. Cultivars resistant to multiple diseases are scarce since breeding for multiple disease resistance is a difficult and lengthy task (https://www.researchgate. net/publication/265820772). Luckily, eleven barley genotypes possessing multiple disease (leaf rust, net blotch and BYDV) resistance were successfully identified in the present study. Such multiple disease resistance is a desirable trait of good agricultural importance to be incorporated in elite barley genotypes through backcross breeding. In most cases, these results were hardly attainable, and signifies the great diversity of, and characteristic feature and quality of, Ethiopian barley landrace collections. Thus, barley breeders should take advantage of this opportunity in producing multi-disease resistant/tolerant barley genotypes in an agronomically enhanced genetic background in Ethiopia and beyond.

Acknowledgments We thank ICARDA and Holetta Agricultural Biotechnology Centre (Ethiopia) for allowing us to use the Virology and Molecular Biology Laboratories. Special thanks go to Professor Tesfaye Mengiste, Purdue University, USA for kindly providing Markers and DNA extraction kits for laboratory analysis done in Ethiopia. The authors thank Ms. Shaimaa Mahmoud and Nourhan Fouad at ICARDA's Biotechnology Laboratory, Cairo, Egypt for excellent technical support. We are grateful to Ethiopian Biodiversity Institute (EBI) and Holetta Agricultural Research Centre for providing barley genotypes.

Funding Field and greenhouse screening work was supported by the Ethiopian Institute of Agricultural Research (EIAR) and East African Agricultural Productivity Project (EAAPP). Molecular laboratory work was supported by ICARDA through Austria Development Agency funded project (Reference No. 2012/05).

\section{Compliance with ethical standards}

Conflict of interest The authors declare that they have no conflict of interest.

Human and animal rights This research does not contain any studies with human participants or animals performed by any of authors.

Ethical approval The authors bear all the ethical responsibilities of this manuscript.

\section{References}

Abebe, D. (2006). Regional strategy for the ex situ conservation of plant genetic resources: Eastern Africa. Eastern Africa Plant Genetic Resources Network (EAPGRN), Entabe, Uganda, July 2006.
Adugna, A. (2011). Barley genetic resources collection and conservation in Ethiopia. In: B. Mulatu \& S. Grando (Eds), Barley Research and Development in Ethiopia. Proceedings of the $2^{\text {nd }}$ National Barley Research and Development Review Workshop. 28-30 November 2006, HARC, Holetta, Ethiopia. ICARDA, Aleppo, Syria pp xiv +391 .

Agranovsky, A. A. (1986). Barley yellow dwarf in Central Ethiopia in 1984/85 and 1985/86 crop seasons. In: Proceedings of the $11^{\text {th }}$ annual meeting of Ethiopian Phytopathological Committee (EPC), 6-7 February 1986 (pp. 56-60). Holetta, Ethiopia.

Agranovsky, A. A., Anisimov, B. V., \& Lister, R. M. (1985). Barley yellow dwarf in Central Ethiopia. In Proceedings of the Regional CIMMYT Wheat Workshop for Eastern, Central and Southern Africa (pp. 141-145). Kenya: Nairobi - Njoro September, 1985.

Ayele, B., Eshetu B., Berhanu B., Bekele H., Melaku D., Asnaketch T., Melkamu A., Amare A., Kiros M., \& Fekede, A. (2008). Review of two decades of research on diseases of small cereal Crops In: Abraham Tadesse (Ed), Increasing Crop Production through Improved Plant Protection - Volume I. Proceedings of the $14^{\text {th }}$ Annual Conference of the Plant Protection Society of Ethiopia (PPSE), 19-22 December 2006. Addis Ababa, Ethiopia. PPSE and EIAR, Addis Ababa, Ethiopia. 598 pp.

Bekele, B. (1998). Distribution of Barley Yellow Dwarf Virus (BYDV) isolates in Arsi and Shewa regions of Ethiopia, and their impact on yield losses. MSc Thesis, Alemaya University, Ethiopia. 168 pp.

Bekele, B., Makkouk, K. M., Yusuf, A., Alemayehu, F., \& Lencho, A. (2001). Occurrence and distribution of barley yellow dwarf virus (BYDV) isolates in Central Ethiopia. International Journal of Pest Management, 47(2), 115-119. https://doi.org/10.1080/09670870151130570.

Bekele, B., Yusuf, A., \& Makkouk, K. M. (2003). Status of barley yellow dwarf and cereal yellow dwarf viruses infecting barley in Ethiopia. Pest Management Journal of Ethiopia, 7, 29-40.

Burnett, P. A., Comeau, A., \& Qualset, C. O. (1995). Host plant tolerance or resistance for control of barley yellow dwarf. In C. J. D'Arcy \& P. A. Burnett (Eds.), Barley yellow dwarf: Forty years of progress (pp. 321-343). St. Paul: APS Press.

Central Statistical Agency (CSA). (2016). Agricultural sample survey 2015/20016 (2008 E.C). In Volume V. Statistical Bulletin 578. CSA, Addis Ababa: Ethiopia.

Collins, N. C., Paltridge, N. G., Ford, C. M., \& Symons, R. H. (1996). The Yd2 gene for barley yellow dwarf virus resistance maps close to the centromere on the long arm of barley chromosome 3. Theoretical and Applied Genetics, 92(7), 858-864. https://doi.org/10.1007/BF00221898.

Doufour, O. A., Palloix, K. G., Salassi, K. G., Pochard, E., \& Marchoux, G. (1989). The distribution of cucumber mosaic virus in resistant and susceptible plants of pepper. Canadian Journal of Botany, 67(3), 655-660. https://doi.org/10.1139 /b89-088.

Doyle, J. J., \& Doyle, J. L. (1990). Isolation of plant DNA from fresh tissue. Focus, 12(1), 13-15.

Dudley, J. W. (1993). Molecular markers in plant improvement: Manipulation of genes affecting quantitative traits. Crop Science, 33(4), 660-668. https://doi.org/10.2135 /cropsci1993.0011183X003300040003x. 
Ford, C. M., Paltridge, N. G., Rathjen, J. P., Moritz, R. I., Simpson, R. J., \& Symons, R. H. (1998). Rapid and informative assays for $\mathrm{Yd} 2$, the barley yellow dwarf virus resistance gene, based on the nucleotide sequence of a closely linked gene. Molecular Breeding, 4(1), 23-31. https://doi.org/10.1023 /A:1009686932457.

Harlan, J. R. (1969). Ethiopia: A center of diversity. Economic Botany, 23(4), 309-314. https://doi.org/10.1007 /BF02860676.

Hayes, J. D., Catherall, P. L., \& Jones, A.T. (1971). Problems encountered in developing BYDV tolerant European cultivars of barley. In Proceedings Second International Barley Genetics Symposium (pp. 493-499). Pullman, Washington, USA.

International Plant Genetic Resources Institute (IPGRI). (1994). Descriptors for barley (Hordeum vulgare L.). In International plant genetic resources institute (IPGRI). Italy: Rome.

Jefferies, S. P., King, B. J., Barr, A. R., Warner, P., Logue, S. J., \& Langridge, P. (2003). Marker-assisted backcross introgression of the $\mathrm{Yd} 2$ gene conferring resistance to barley yellow dwarf virus in barley. Plant Breeding, 122(1), 52-56. https://doi.org/10.1046/j.1439-0523.2003.00752.x.

Jensen, S. G. (1973). Systemic movement of barley yellow dwarf virus in small grains. Phytopathology, 63(7), 854-856. https://doi.org/10.1094/Phyto-63-854.

Jones, A. T., \& Catherall, P. L. (1970). The relationship between growth rate and the expression of the tolerance to barley yellow dwarf virus in barley. Annals of Applied Biology, 65(1), 137-145. https://doi.org/10.1111/j.1744-7348.1970. tb04571.x.

Korzun, V. (2003). Molecular markers and their applications in cereals breeding. A paper presented during the FAO international workshop on "marker assisted selection: A fast track to increase genetic gain in plant and animal breeding?". 1718 October 2003. Italy: Turin.

Law, M. D., Moyer, J. W., \& Payne, G. A. (1989). Effect of the host resistance on pathogenesis of maize dwarf mosaic virus. Phytopathology, 79(7), 757-761. https://doi.org/10.1094 /phyto-79-757.

Makkouk, K. M., \& Comeau, A. (1994). Evaluation of various methods for the detection of barley yellow dwarf luteovirus by the tissue-blot immunoassay and its use for BYDV detection in cereals inoculated at different growth stages. European Journal of Plant Pathology, 100(1), 71-80. https://doi.org/10.1007/BF01871967.

Makkouk, K. M., \& Ghulam, W. (1992). Resistance of barley genotypes with $\mathrm{Yd} 2$ gene to the movement of barley yellow dwarf virus. Rachis Newsletter, 11(1/2), 81-82.

Makkouk, K. M., Comeau, A., \& ST-Pierre, C. A. (1994). Screening for barley yellow dwarf luteovirus resistance in barley on the basis of virus movement. Journal of Phytopathology, 141(2), 165-172. https://doi.org/10.1111 /j.1439-0434.1994.tb01458.x.

Niks, R. E., Habekuss, A., Bekele, B., \& Ordon, F. (2004). A novel major gene on chromosome $6 \mathrm{H}$ for resistance of barley against the barley yellow dwarf virus. Theoretical and Applied Genetics, 109(7), 1536-1543. https://doi. org/10.1007/s00122-004-1777-7.

Nono-Womdim, R., Marchoux, E., Pochard, E., Palloix, A., \& Gebreselassie, K. (1991). Resistance of pepper lines to the movement of cucumber mosaic virus. Journal of
Phytopathology, 132(1), 21-32. https://doi.org/10.1111 /j.1439-0434.1991.tb00090.x.

Paltridge, N. G., Collins, N. C., Bendahmane, A., \& Symons, R. H. (1998). Development of YLM, a co-dominant PCR marker closely linked to the $Y d 2$ gene for resistance to barley yellow dwarf disease. Theoretical and Applied Genetics, 96(8), 1170-1177. https://doi.org/10.1007/s001220050853.

Paterson, A. H., Tanksley, S. D., \& Sorrells, M. E. (1991). DNA markers in crop improvement. In D. L. Sparks (Ed.), Advances in agronomy (pp. 39-90). New York: Academic Press.

Peterson, R. F., Campbell, A. B., \& Hannah, A. E. (1948). A diagrammatic scale for estimating rust intensity on leaves and stems of cereals. Canadian Journal of Research, 26c(5), 496-500. https://doi.org/10.1139/cjr48c-033.

Plant Protection Research Centre (PPRC). (2012). Progress report for the period of 2010-2011. Ambo, Ethiopia: PPRC.

Qualset, C. O. (1975). Sampling germplasm in a center of diversity: An example of disease resistance in Ethiopian barley. In O. H. Fraenkel \& J. W. Hawkes (Eds.), Crop Genetic Resources for Today and Tomorrow (pp. 81-98). Cambridge University Pres.

Ramsay, L., Macaulay, M., Ivanissevich, S. D., MacLean, K., Cardle, L., Fuller, J., Edwards, K. J., Tuvesson, S., Morgante, M., Massari, A., Maestri, E., Marmiroli, N., Sjakste, T., Ganal, M., Powell, W., \& Waugh, R. (2000). A simple sequence repeat-based linkage map of barley. Genetics, 156(4), 1997-2005.

Rasmusson, D. C., \& Schaller, C. W. (1959). The inheritance of resistance in barley yellow dwarf virus. Agronomy Journal, 51, 661-664. https://doi. org/10.2134/agronj1959.00021962005100110009x.

Schaller, C. W., \& Qualset, C. O. (1980). Breeding for resistance to the barley yellow dwarf virus. In Proceedings, Third International Wheat Conference, Madrid, Spain. University of Nebraska Agricultural Experiment Station publication MP41, 528-541.

Spaner, D., Shugar, L. P., Choo, T. M., Falak, I., Briggs, K. G., Legge, W. G., Falk, D. E., Ullrich, S. E., Tinker, N. A., Steffenson, B. J., \& Mather, D. E. (1998). Mapping of disease resistance loci in barley on the basis of visual assessment of naturally occurring symptoms. Crop Science, 38(3), 843850. https://doi.org/10.2135/cropsci1998.0011183 X003800030037x.

Stuber, C. W., Polacco, M., \& Senior, M. L. (1999). Synergy of empirical breeding, marker-assisted selection, and genomics to increase yield potential. Crop Science, 39(6), 1571-1583. https://oi.org/10.2135/cropsci1999.3961571x.

Vavilov, N. R. (1951). The origin, variation, immunity and breeding of cultivated plants. Chronica Botanica, 13(1/6), 1-366. https://doi.org/10.1126/science.115.2990.433-a.

Yusuf, A., Makkouk, K. M., Beniwal, S. P. S., \& Semeane, Y. (1992). Survey of Barley Yellow Dwarf Virus in small-grain cereals in the Ethiopian Highlands. In A. Comeau \& K. M. Makkouk (Eds.), Barley yellow dwarf in West Asia and North Africa: Proceedings of a workshop (pp. 87-90). Rabat, Morocco: ICARDA. 19-21 November 1989.

Zadoks, J. C., Chang, T. T., \& Konzak, C. F. (1974). A decimal code for the growth stages of cereals. Weed Research, 14(6), 415-421. https://doi.org/10.1111/j.1365-3180.1974.tb01084. 